\title{
Prestress Effect on the Thermomechanical Response of Viscoelastic Plate Under Harmonic Loading
}

\author{
Oleksandr Ostos ${ }^{*}$, Yaroslav Zhuk \\ Faculty of Mechanics and Mathematics, Taras Shevchenko National University of Kyiv, Kyiv, Ukraine \\ Email address: \\ y.zhuk@i.ua (Y.Zhuk), ostos.alexander1994@gmail.com (O. Ostos) \\ ${ }^{*}$ Corresponding author
}

To cite this article:

Oleksandr Ostos, Yaroslav Zhuk. Prestress Effect on the Thermomechanical Response of Viscoelastic Plate Under Harmonic Loading. Advances in Applied Sciences. Vol. 5, No. 2, 2020, pp. 20-27. doi: 10.11648/j.aas.20200502.11

Received: March 23, 2020; Accepted: April 13, 2020; Published: April 23, 2020

\begin{abstract}
A statement of the coupled thermomechanical problem on forced resonant vibrations and dissipative heating of hinged viscoelastic elastomeric plate is given with account of prestresses present in the plate. It is assumed the prestress is generated as a result of the manufacturing process or preliminary plate service. The problem statement is based on the standard Kirchhoff-Love hypotheses and concept of complex moduli that are used to describe the viscoelastic material response to harmonic loading. Under these circumstances, the prestress manifests itself as a membrane forces applied in the plane of the rectangular plate. Therefore, the problem of in-plane stress state and problem of forced transverse vibration of the plate can be solved separately. Both steady-state and transient thermal response is investigated. Influence of the prestress is studied in details. Dissipative heating temperature histories are calculated for the variety of the prestress and loading parameters. Temperature criterion is adopted to determine the critical state. The data obtained are used for the plate fatigue life prediction as well as for the investigation of prestress effect on the plate response. The reliability of the values of frequencies on the several lowest resonances was checked. For the most energy-intensive first mode of transverse vibrations, the influence of the preliminary tensile stress state, as well as the amplitude of the transverse distributed load on the amplitude-frequency characteristics and temperature evolution was studied.
\end{abstract}

Keywords: Thermomechanical Coupling, Viscoelastic Plate, Complex Moduli, Prestress, Dissipative Heating

\section{Introduction}

Thermomechanical coupling problems are common phenomena in the field of Solid Mechanics. Mechanical engineering equipment [1], aerospace industry structures and energy generation machinery as well as transport engine/structure components can be subjected to severe mechanical and thermal cyclic loads. Assessment of component life depends on the behavior of materials used. Elastomers are widely used as materials for the structural components mentioned. Under intensive cyclic loading, the effect of dissipative heating can be particularly profound for the polymers. The dissipative heating is caused by an appearance of a mechanical hysteresis resulting from the viscoelastic nature of the most of industrial polymers. Part of the dissipated mechanical energy leads to generation of thermal energy [2-5], while the rest of the energy transferred to the structure is both partly stored in the material [6] and dissipated as a result of plastic deformation [7, 8], viscoplastic deformation, and variations of yield limit caused by isotropic and kinematic hardening [9].

It is generally adopted that dissipative heating is usually attributed to the internal friction of the material [10], which causes the hysteretic response. Energy dissipated as a heat leads to the increase of the temperature of the loaded structure. Small temperature advance over one separate cycle can result in high heating level for prolonged operational period [11, 12].

The effect of thermomechanical coupling described above should be taken account for when the adequate models for fatigue life predictions are developed. This implies a necessity for the deep investigation of dissipative heating, including its physical nature, the consequences, and the relations with degradation mechanisms in structural elements, as well as the estimation of safe temperature ranges.

Additional factor that can significantly influence the fatigue life prediction of the polymeric structures is the residual stress 
which can arise as a result of manufacturing processes. For the subsequent cyclic loading problem, these stresses should be considered as prestresses. The effect of the prestresses, as well, has to be taken into account to improve the reliability of the fatigue life prediction technique.

To address the issue of fatigue life prediction with account of thermomechanical coupling, the theories based on the relations between the amplitudes of main field variables are usually used to describe the thermomechanical response of viscoelastic solids under cyclic loading. Application of complex amplitudes and complex moduli concept in the case of harmonic loading yields the complete system of amplitude equations for linearized coupled thermoviscoelasticity in terms of complex amplitudes. This system is complemented with the complex analogues of the equation of motion along with the stationary or non-stationary equation of heat transfer. In the former case, the temperature-frequency characteristics of vibration can be obtained and the critical loading parameters can be specified. To describe the over critical heating and, therefore, to build the fatigue $S-N$ curves the latter equation should be used instead. The detailed reviews of the theories and approaches developed along with the numerous applications can be found in [13-18].

In this paper, a fatigue model for prediction of a number of cycles to thermal failure is developed, taking into consideration stationary dissipative heating and structure prestress that can significantly affect the fatigue life characteristics of the viscous rectangular plate subjected to transverse harmonic loading. Special attention is paid to the influence of membrane prestress on the amplitude-frequency and temperature-frequency characteristics as well as on the non-stationary histories of dissipative heating for cyclically excited plate.

\section{Problem Statement}

\subsection{Theoretical Concepts}

Let us consider a hingedly supported plate of thickness $h$ with planform dimensions $a$ and $b$. It is assumed that the plate is composed of the linear viscoelastic material which material properties are frequency, amplitude and temperature independent. The rectangular Cartesian coordinates $(x, y, z)$ with the $x y$-plane coinciding with the geometric middle plane of the plate and the $z$-coordinate taken positive upward is introduced. The plate contour is formed by the edges $x=0, a$; $y=0, b$. It is also assumed that prestresses are generated in the plate as a result of manufacturing process. The prestresses are simulated by the presence of normal membrane forces $N_{x x}$, $N_{y y}$ and, in general case, by shear membrane force $N_{x y}$. The plate is subjected to transverse loading

$$
q_{z}=q_{0}^{\prime}(x, y) \cos \omega t-q_{0}^{\prime \prime}(x, y) \sin \omega t,
$$

that is distributed over the plate surface according to specific law and is harmonically varying in time $t$ with amplitude

$$
q_{0}(x, y)=\left(q_{0}^{\prime}(x, y)^{2}+q_{0}^{\prime \prime}(x, y)^{2}\right)^{1 / 2}
$$

and frequency $\omega$ which is close to one of the resonances.

The plate edges are assumed to be thermally insulated while the convective heat transfer to the ambient medium of temperature $T_{C}$ is supposed to take place at the face surfaces of the plate.

To simulate the mechanical response of the plate under consideration, the classical Kirchhoff-Love hypotheses are made use of $[19,20]$. The integral operators of linear viscoelasticity are used to describe the viscoelastic properties of the plate material [13-15]. In the particular case of harmonic deformation, these operators are reduced to multiplication of complex quantities

$$
\tilde{a} * \tilde{b}=\left(a^{\prime}+i a^{\prime \prime}\right)\left(b^{\prime}+i b^{\prime \prime}\right),
$$

where the operator and complex quantities are marked with asterisk and wave respectively. It is also assumed that temperature is constant through the plate thickness.

\subsection{Main Equations}

In general case, the equations of transverse vibration of the plate are obtained in the following form [19, 20]

$$
\left\{\begin{array}{r}
\frac{\partial N_{x x}}{\partial x}+\frac{\partial N_{x y}}{\partial y}=J_{0} \frac{\partial^{2} u}{\partial t^{2}}, \\
\frac{\partial N_{x y}}{\partial x}+\frac{\partial N_{y y}}{\partial y}=J_{0} \frac{\partial^{2} v}{\partial t^{2}}, \\
\frac{\partial^{2} M_{x x}}{\partial x^{2}}+2 \frac{\partial^{2} M_{x y}}{\partial x \partial y}+\frac{\partial^{2} M_{y y}}{\partial y^{2}}+N(u, v, w)-k w+q(x, y, t)= \\
=J_{0} \frac{\partial^{2} w}{\partial t^{2}}-J_{2} \frac{\partial^{2}}{\partial t^{2}}\left(\frac{\partial^{2} w}{\partial x^{2}}+\frac{\partial^{2} w}{\partial y^{2}}\right),
\end{array}\right.
$$

where $u, v$ and $w$ are components of the displacement field along $O x, O y$ and $O z$ axis respectively; $N_{i j}$ and $M_{i j}$, $i, j=x, y$ are thickness-integrated forces and moments, known as the stress resultants $[19,20]$;

$$
N(u, v, w)=\frac{\partial}{\partial x}\left(N_{x x} \frac{\partial w}{\partial x}+N_{x y} \frac{\partial w}{\partial y}\right)+\frac{\partial}{\partial y}\left(N_{x y} \frac{\partial w}{\partial x}+N_{y y} \frac{\partial w}{\partial y}\right)
$$

$k$ is the foundation modulus (for the cases where it has to be taken account for), $\rho$ is the material density.

In system (4), $J_{0}$ and $J_{2}$ are the mass moments of inertia

$$
J_{0}=\int_{-h / 2}^{h / 2} \rho d z, J_{2}=\int_{-h / 2}^{h / 2} \rho z^{2} d z
$$

and the term with $J_{2}$ should be preserved if the rotary inertia effects are of particular interest.

\subsubsection{Equation of Motion and Boundary Conditions}

As the Kirchhoff-Love hypotheses are considered to be valid so the membrane and bending stresses are appeared to be 
uncoupled. It is also assumed the prestresses induced are constant $\quad\left(N_{x x}=N_{x x}^{0}=\right.$ const,$N_{y y}=N_{y y}^{0}=$ const , $N_{x y}=N_{x y}^{0}=$ const ). Under these assumptions, the in-plane problem for the plate becomes statically determinate. Thus, the first and second equations from (4) are satisfied identically.

Classical plate theory formalism for isotropic elastic material yields the $3^{\text {rd }}$ equation from (4) in the form

$$
D \nabla^{4} w+\rho h \frac{\partial^{2} w}{\partial t^{2}}=N_{x x}^{0} \frac{\partial^{2} w}{\partial x^{2}}+2 N_{x y}^{0} \frac{\partial^{2} w}{\partial x \partial y}+N_{y y}^{0} \frac{\partial^{2} w}{\partial y^{2}}+q(x, y, t),
$$

where $D=E h^{3} / 12\left(1-v^{2}\right)$ is the plate bending stiffness coefficient, $E$ and $v$ are the material Young's modulus and Poisson ratio respectively;

$$
\nabla^{4}=\frac{\partial^{4}}{\partial x^{4}}+2 \frac{\partial^{4}}{\partial x^{2} \partial y^{2}}+\frac{\partial^{4}}{\partial y^{4}}
$$

As a result, in the case of temperature independent material properties and assumption of a temperature constancy through the plate thickness, the mechanical problem is reduced to two uncoupled problems: (i) plane stress problem, (ii) pure bending problem. The former one defines the prestress while the latter problem describes the varying harmonically in time pure bending state which is superimposed over the prestressed state of the plate.

Boundary conditions for the linear bending of a rectangular plate with edges parallel to the $x$ and $y$ coordinates for the particular case of hingedly supported have the form

$$
w=0, \frac{\partial^{2} w}{\partial x^{2}}=0 \text { at } x=0, a ; \quad w=0, \frac{\partial^{2} w}{\partial y^{2}}=0 \text { at } y=0, b .
$$

Under harmonic loading, investigation of steady-state vibration regime is usually performed with the use of complex moduli and complex amplitudes concept [13-16]. These yields the equation (7) as follows

$$
\tilde{D} \nabla^{4} \tilde{w}+\rho h \omega^{2} \frac{\partial^{2} \tilde{w}}{\partial t^{2}}=N_{x x}^{0} \frac{\partial^{2} \tilde{w}}{\partial x^{2}}+2 N_{x y}^{0} \frac{\partial^{2} \tilde{w}}{\partial x \partial y}+N_{y y}^{0} \frac{\partial^{2} \tilde{w}}{\partial y^{2}}+\tilde{q}(x, y),
$$

where $\tilde{D}=D^{\prime}+i D^{\prime \prime}, \quad \tilde{w}(x, y)=w^{\prime}(x, y)+i w^{\prime \prime}(x, y), \quad$ and $\tilde{q}(x, y)=q^{\prime}(x, y)+i q^{\prime \prime}(x, y)$ are complex bending stiffness coefficient, deflection of the plate and load; $i=\sqrt{-1}$. It is worth mentioning here that expressions for the boundary conditions should have the same form (9) with substitution of complex variable $\tilde{w}$ instead of real one $w$.

To obtain the time variation laws for the beam deflection and load, one should apply the formulas

$$
\begin{aligned}
& w(x, y, t)=w^{\prime}(x, y) \cos \omega t-w^{\prime \prime}(x, y) \sin \omega t, \\
& q(x, y, t)=q^{\prime}(x, y) \cos \omega t+i q^{\prime \prime}(x, y) \sin \omega t .
\end{aligned}
$$

Here $(\cdot)^{\prime}$ and $(\cdot)^{\prime \prime}$ are the real and imaginary parts of the complex amplitude.

The complex equation (10) is equivalent to system of two real equations with respect to the complex amplitudes in the form

$$
\left\{\begin{array}{l}
D^{\prime} \nabla^{4} w^{\prime}-D^{\prime \prime} \nabla^{4} w^{\prime \prime}-\rho h \omega^{2} w^{\prime}=N_{x x}^{0} \frac{\partial^{2} w^{\prime}}{\partial x^{2}}+2 N_{x y}^{0} \frac{\partial^{2} w^{\prime}}{\partial x \partial y}+N_{y y}^{0} \frac{\partial^{2} w^{\prime}}{\partial y^{2}}+q^{\prime}, \\
D^{\prime} \nabla^{4} w^{\prime \prime}+D^{\prime \prime} \nabla^{4} w^{\prime}-\rho h \omega^{2} w^{\prime \prime}=N_{x x}^{0} \frac{\partial^{2} w^{\prime \prime}}{\partial x^{2}}+2 N_{x y}^{0} \frac{\partial^{2} w^{\prime \prime}}{\partial x \partial y}+N_{y y}^{0} \frac{\partial^{2} w^{\prime \prime}}{\partial y^{2}}+q^{\prime \prime}
\end{array}\right.
$$

with the boundary conditions (9) rewritten in terms of the complex amplitudes as well

$$
\begin{aligned}
& w^{\prime}=w^{\prime \prime}=0, \frac{\partial^{2} w^{\prime}}{\partial x^{2}}=\frac{\partial^{2} w^{\prime \prime}}{\partial x^{2}}=0 \text { at } x=0, a \\
& w^{\prime}=w^{\prime \prime}=0, \frac{\partial^{2} w^{\prime}}{\partial y^{2}}=\frac{\partial^{2} w^{\prime \prime}}{\partial y^{2}}=0 \text { at } y=0, b .
\end{aligned}
$$

\subsubsection{The Dissipative Function}

Evolution of the temperature field is described by the heat conduction equation averaged over the plate thickness and vibration period

$$
\rho \operatorname{ch} \frac{\partial \theta}{\partial t}=\lambda h\left(\frac{\partial^{2} \theta}{\partial x^{2}}+\frac{\partial^{2} \theta}{\partial y^{2}}\right)-2 \alpha_{S} \theta+\left\langle\bar{W}_{D}\right\rangle
$$

complemented with thermal initial and boundary conditions

$$
\theta=0 \text { at } t=0 ; \frac{\partial \theta}{\partial x}=0 \text { at } x=0, a ; \frac{\partial \theta}{\partial y}=0 \text { at } y=0, b \text {. }
$$

In the equations (14) and (15), $\theta$ is the dissipative heating temperature, $\theta=T-T_{C} ; c, \lambda$ and $\alpha_{S}$ are the specific heat, heat conductivity coefficient and heat transfer coefficient at the face surfaces of the plate respectively; $T_{C}$ is the ambient temperature; $\left\langle\bar{W}_{D}\right\rangle$ is the dissipative function averaged over the period of vibrations and over the plate thickness

$$
\begin{aligned}
& \left\langle\bar{W}_{D}\right\rangle=\frac{\omega}{2}\left[\left(M_{x x}^{\prime \prime} \varepsilon_{x x}^{1^{\prime}}-M_{x x}^{\prime} \varepsilon_{x x}^{1 \prime \prime}\right)+\left(M_{y y}^{\prime \prime} \varepsilon_{y y}^{1^{\prime}}-M_{y y}^{\prime} \varepsilon_{y y}^{1 \prime \prime}\right)+\right. \\
& \left.+\left(M_{x y}^{\prime \prime} \gamma_{x y}^{1}{ }^{\prime}-M_{x y}^{\prime} \gamma_{x y}^{1 \prime \prime}\right)\right] ;
\end{aligned}
$$

where

$$
\begin{gathered}
\tilde{\varepsilon}_{x x}^{1}=\varepsilon_{x x}^{1^{\prime}}+i \varepsilon_{x x}^{1 \prime \prime}=-\frac{\partial^{2} \tilde{w}}{\partial x^{2}}, \tilde{\varepsilon}_{y y}^{1}=\varepsilon_{y y}^{1}+i \varepsilon_{y y}^{\prime \prime}=-\frac{\partial^{2} \tilde{w}}{\partial y^{2}}, \\
\tilde{\gamma}_{x y}^{1}=\gamma_{x y}^{1 \prime}+i \gamma_{x y}^{\prime \prime \prime}=-2 \frac{\partial^{2} \tilde{w}}{\partial x \partial y} ; \\
\tilde{M}_{x x}=M_{x x}^{\prime}+i M_{x x}^{\prime \prime}=-\tilde{D}\left[\frac{\partial^{2} \tilde{w}}{\partial x^{2}}+\tilde{v} \frac{\partial^{2} \tilde{w}}{\partial y^{2}}\right] ; \\
\tilde{M}_{y y}=M_{y y}^{\prime}+i M_{y y}^{\prime \prime}=-\tilde{D}\left[\tilde{\mathrm{v}} \frac{\partial^{2} \tilde{w}}{\partial x^{2}}+\frac{\partial^{2} \tilde{w}}{\partial y^{2}}\right]
\end{gathered}
$$




$$
\tilde{M}_{x y}=M_{x y}^{\prime}+i M_{x y}^{\prime \prime}=-\tilde{D}(1-\tilde{v}) \frac{\partial^{2} \tilde{w}}{\partial x \partial y} .
$$

In the expressions (17), the quantities $\tilde{M}_{i j}, \tilde{\varepsilon}_{i j}^{1}$, and $\tilde{\gamma}_{x y}^{1}$, $i, j=x, y$ are complex-value analogues of moments from equations (4) and bending strains (curvatures and twisting); $\tilde{v}$ is the complex Poisson ratio, $\tilde{v}=v^{\prime}-i v^{\prime \prime}$. Let us emphasize here that version of the cyclic viscoelasticity with real Poisson ratio and complex bulk modulus is chosen for the materials under consideration in this problem. Thus, relations $v^{\prime}=v$, $v^{\prime \prime}=0$ are assumed to be valid further on.

As a result, a statement of the coupled thermomechanical problem on forced vibrations and dissipative heating of hinged viscoelastic plate with account of prestresses applied is given by the equations (12) and (14) with the expression (16) for the dissipative function complemented with correspondent mechanical boundary conditions (13) along with thermal initial and boundary conditions (15).

\section{Solution Technique}

Let us study the case when the prestress state can be a result of presence of the normal preliminary traction only: $N_{x x}^{0}=$ const $\neq 0, N_{y y}^{0}=0, N_{x y}^{0}=0$.

It is worth mentioning here that for cyclically stable materials, mechanical processes stabilize much faster than the thermal ones [13]. It enables us to consider mechanical part of the problem as the steady-state process in contrast to the thermal part which is studied in the frame of transient analysis at the aforementioned assumptions with respect to the material constants. Thus, solution of the steady state vibration problem for the hingedly supported plate can be written in the form $[13,20]$

$$
\begin{aligned}
& w^{\prime}(x, y)=\sum_{m, n=1}^{\infty} w_{m n}^{\prime} \sin \frac{m \pi x}{a} \sin \frac{n \pi y}{b}, \\
& w^{\prime \prime}(x, y)=\sum_{m, n=1}^{\infty} w_{m n}^{\prime \prime} \sin \frac{m \pi x}{a} \sin \frac{n \pi y}{b},
\end{aligned}
$$

where $w_{m n}^{\prime}$ and $w_{m n}^{\prime \prime}$ are unknown complex amplitudes of the eigenmode $m n$ corresponding to the natural frequency $\omega_{m n}$. This frequency is determined by the well-known formula taking account for the prestresses $[13,20]$

$$
\omega_{m n}=\sqrt{\frac{1}{\rho h}\left\{D_{0}\left[\left(\frac{m \pi}{a}\right)^{2}+\left(\frac{n \pi}{b}\right)^{2}\right]^{2}+\left(\frac{m \pi}{a}\right)^{2} N_{x x}^{0}\right\}},
$$

where $D_{0}$ is the plate bending stiffness coefficient calculated for elastic response of the plate material. In the case of viscoelastic material, it should be calculated as $D_{0}^{2}=D^{2}+D^{\prime \prime 2}$.

Vibration of a plate in the vicinity of the resonance frequency $\omega=\omega_{m n}$ is under consideration in the present study. It is also assumed that the loading is distributed over the plate surface according to the law corresponding to this particular mode of vibration to facilitate the resonant vibration

$$
\begin{aligned}
& q(x, y, t)=q_{m n}^{0 \prime} \sin \frac{m \pi x}{a} \sin \frac{n \pi y}{b} \cos \omega t- \\
& -q_{m n}^{0 \prime \prime} \sin \frac{m \pi x}{a} \sin \frac{n \pi y}{b} \sin \omega t .
\end{aligned}
$$

If the transverse loading varies according to sine or cosine law over the time then this relation can be modified in the obvious way.

In this case, the plate response will be formed by the resonating mode $m n$, and the sums in the expression (18) can be discarded. Substitution of the resultant into (17) with accounting for $N_{x y}^{0}=0$ yields the system of two linear algebraic equations with respect to $w_{m n}^{\prime}$ and $w_{m n}^{\prime \prime}$ of the following form

$$
\left\{\begin{array}{l}
w_{m n}^{\prime}\left[D^{\prime} A-\rho h \omega^{2}+B\right]-w_{m n}^{\prime \prime} D^{\prime \prime} A=q_{m n}^{\prime}, \\
w_{m n}^{\prime} D^{\prime \prime} A+w_{m n}^{\prime \prime}\left[D^{\prime} A-\rho h \omega^{2}+B\right]=q_{m n}^{\prime \prime},
\end{array}\right.
$$

where $A=k_{x}^{4}+2 k_{x}^{2} k_{y}^{2}+k_{y}^{4}, B=N_{x x}^{0} k_{x}^{2}+N_{y y}^{0} k_{y}^{2} ; k_{x}=m \pi x / a$, $k_{y}=n \pi x / b$.

Solution of the system of equations (21) is determined by the expression

$$
w_{m n}^{\prime}=\Delta_{1} / \Delta, \quad w_{m n}^{\prime \prime}=\Delta_{2} / \Delta ;
$$

where

$$
\begin{aligned}
& \Delta_{1}=q_{m n}^{\prime}\left[D^{\prime}\left(k_{x}^{4}+2 k_{x}^{2} k_{y}^{2}+k_{y}^{4}\right)-\rho h \omega^{2}+\left(N_{x x}^{0} k_{x}^{2}+N_{y y}^{0} k_{y}^{2}\right)\right]+ \\
& +q_{m n}^{\prime \prime} D^{\prime \prime}\left(k_{x}^{4}+2 k_{x}^{2} k_{y}^{2}+k_{y}^{4}\right), \\
& \Delta_{2}=q_{m n}^{\prime \prime}\left[D^{\prime}\left(k_{x}^{4}+2 k_{x}^{2} k_{y}^{2}+k_{y}^{4}\right)-\rho h \omega^{2}+\left(N_{x x}^{0} k_{x}^{2}+N_{y y}^{0} k_{y}^{2}\right)\right]- \\
& -q_{m n}^{\prime} D^{\prime \prime}\left(k_{x}^{4}+2 k_{x}^{2} k_{y}^{2}+k_{y}^{4}\right), \\
& \Delta=\left[D^{\prime}\left(k_{x}^{4}+2 k_{x}^{2} k_{y}^{2}+k_{y}^{4}\right)-\rho h \omega^{2}+\left(N_{x x}^{0} k_{x}^{2}+N_{y y}^{0} k_{y}^{2}\right)\right]^{2}+ \\
& +\left[D^{\prime \prime}\left(k_{x}^{4}+2 k_{x}^{2} k_{y}^{2}+k_{y}^{4}\right)\right]^{2} .
\end{aligned}
$$

To address the transient heat conductivity equation, one should define the expression for dissipative function $\left\langle\bar{W}_{D}\right\rangle$ from (14) as a function of the complex amplitudes of deflection. With making use of the expressions (17), the relations between moment and deflection amplitudes can be derived as follows

$$
\begin{aligned}
& M_{x x}^{\prime}=-D^{\prime}\left[\frac{\partial^{2} w_{m n}^{\prime}}{\partial x^{2}}+v \frac{\partial^{2} w_{m n}^{\prime}}{\partial y^{2}}\right]+D^{\prime \prime}\left[\frac{\partial^{2} w_{m n}^{\prime \prime}}{\partial x^{2}}+v \frac{\partial^{2} w_{m n}^{\prime \prime}}{\partial y^{2}}\right] \\
& M_{x x}^{\prime \prime}=-D^{\prime \prime}\left[\frac{\partial^{2} w_{m n}^{\prime}}{\partial x^{2}}+v \frac{\partial^{2} w_{m n}^{\prime}}{\partial y^{2}}\right]-D^{\prime}\left[\frac{\partial^{2} w_{m n}^{\prime \prime}}{\partial x^{2}}+v \frac{\partial^{2} w_{m n}^{\prime \prime}}{\partial y^{2}}\right]
\end{aligned}
$$




$$
\begin{gathered}
M_{y y}^{\prime}=-D^{\prime}\left[\frac{\partial^{2} w_{m n}^{\prime}}{\partial y^{2}}+v \frac{\partial^{2} w_{m n}^{\prime}}{\partial x^{2}}\right]+D^{\prime \prime}\left[\frac{\partial^{2} w_{m n}^{\prime \prime}}{\partial y^{2}}+v \frac{\partial^{2} w_{m n}^{\prime \prime}}{\partial x^{2}}\right] \\
M_{x x}^{\prime \prime}=-D^{\prime \prime}\left[\frac{\partial^{2} w_{m n}^{\prime}}{\partial y^{2}}+v \frac{\partial^{2} w_{m n}^{\prime}}{\partial x^{2}}\right]-D^{\prime}\left[\frac{\partial^{2} w_{m n}^{\prime \prime}}{\partial y^{2}}+v \frac{\partial^{2} w_{m n}^{\prime \prime}}{\partial x^{2}}\right] ; \\
M_{x y}^{\prime}=-(1-v)\left(D^{\prime} \frac{\partial^{2} w_{m n}^{\prime}}{\partial x \partial y}-D^{\prime \prime} \frac{\partial^{2} w_{m n}^{\prime \prime}}{\partial x \partial y}\right) ; \\
M_{x y}^{\prime \prime}=-(1-v)\left(D^{\prime \prime} \frac{\partial^{2} w_{m n}^{\prime}}{\partial x \partial y}+D^{\prime} \frac{\partial^{2} w_{m n}^{\prime \prime}}{\partial x \partial y}\right) .
\end{gathered}
$$

Substitution of (24) into (16) with accounting for the first line from (17) yields the expression for the dissipative function

$$
\begin{aligned}
& \left\langle\bar{W}_{D}\right\rangle=\frac{\omega}{2} D^{\prime \prime}\left\{\left(\frac{\partial^{2} w_{m n}^{\prime}}{\partial x^{2}}\right)^{2}+\left(\frac{\partial^{2} w_{m n}^{\prime \prime}}{\partial x^{2}}\right)^{2}+\left(\frac{\partial^{2} w_{m n}^{\prime}}{\partial y^{2}}\right)^{2}+\right. \\
& +\left(\frac{\partial^{2} w_{m n}^{\prime \prime}}{\partial y^{2}}\right)^{2}+2 v\left[\frac{\partial^{2} w_{m n}^{\prime}}{\partial x^{2}} \frac{\partial^{2} w_{m n}^{\prime}}{\partial y^{2}}+\frac{\partial^{2} w_{m n}^{\prime \prime}}{\partial x^{2}} \frac{\partial^{2} w_{m n}^{\prime \prime}}{\partial y^{2}}\right]+ \\
& \left.+(1-v)\left[\left(\frac{\partial^{2} w_{m n}^{\prime}}{\partial x \partial y}\right)^{2}+\left(\frac{\partial^{2} w_{m n}^{\prime \prime}}{\partial x \partial y}\right)^{2}\right]\right\} .
\end{aligned}
$$

The transient heat conductivity problem formed by the equation (14) complemented by the expression for the dissipative function (25) along with the thermal initial and boundary conditions (15) can be solved numerically.

\section{Numerical Results and Analysis}

Calculations were performed for rectangular plate with the geometric parameters $a=0.15 \mathrm{~m}, b=0.1 \mathrm{~m}$, and $h=0.005 \mathrm{~m}$. Polyethylene was chosen as the plate material. Complex moduli for the material were taken from [21]: $E^{\prime}=8.0 \times 10^{8} \mathrm{~Pa}$; $E^{\prime \prime}=6.3 \times 10^{6} \mathrm{~Pa} ; \quad \operatorname{tg} \theta=E^{\prime \prime} / E^{\prime}=0.0079$. Polyethylene Poisson ratio and density are chosen to be equal to $v=0.46$ and $\rho=953 \mathrm{~kg} / \mathrm{m}^{3}$ respectively. Thermal parameters for the problem were defined as follows: specific heat $c=2300 \mathrm{~W} /(\mathrm{kg} \cdot$ 癈 $)$, heat conductivity coefficient $\lambda=0.36 \mathrm{~W} /(\mathrm{m} \cdot$ 癈 $)$ and heat transfer coefficient at the face surfaces of the plate $\alpha_{S}=4 \mathrm{~W} /\left(\mathrm{m}^{2}\right.$. 癈 $)$.

Harmonic loading of the plate with $N_{y y}=0$ and different preliminary normal membrane forces $N_{x x}$ was investigated.

\subsection{Stationary Problem}

The stationary problem was first solved.

A natural-vibration frequency in the first eigenmode ( $m=1$, $n=1$ ) of the square plate with $N_{x x}=45 \mathrm{kN} / \mathrm{m}$ under a transverse loading with amplitude $q^{\prime}=400 \mathrm{~Pa}$ (with $q^{\prime \prime}=0$ ) is $f_{11}=468.1 \mathrm{~Hz}$. Since the investigation was carried out in the near-resonance region, the distribution of the deflection and temperature were based on the fundamental frequency $f_{11}$, where $f_{m n}=\omega_{m n} / 2 \pi$ is the natural frequency corresponding to the first mode of vibration. The distribution of the deflection and the temperature of the plate are shown below.

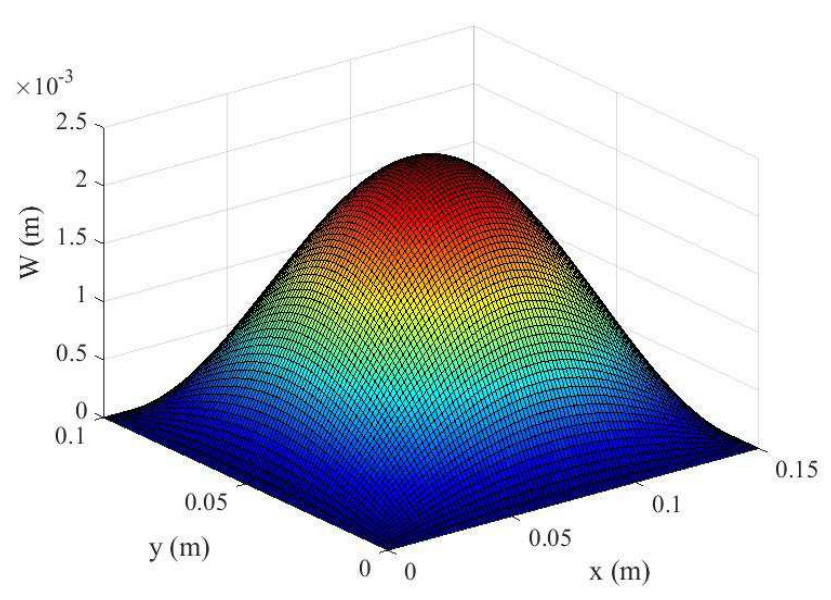

Figure 1. The distribution of the deflection of the plate.

The maximum deflection, $w_{\max }$, is achieved in the center of the square plate point $(x=a / 2, y=b / 2)$ and is equal to $2.4 \times 10^{-3} \mathrm{~m}$.

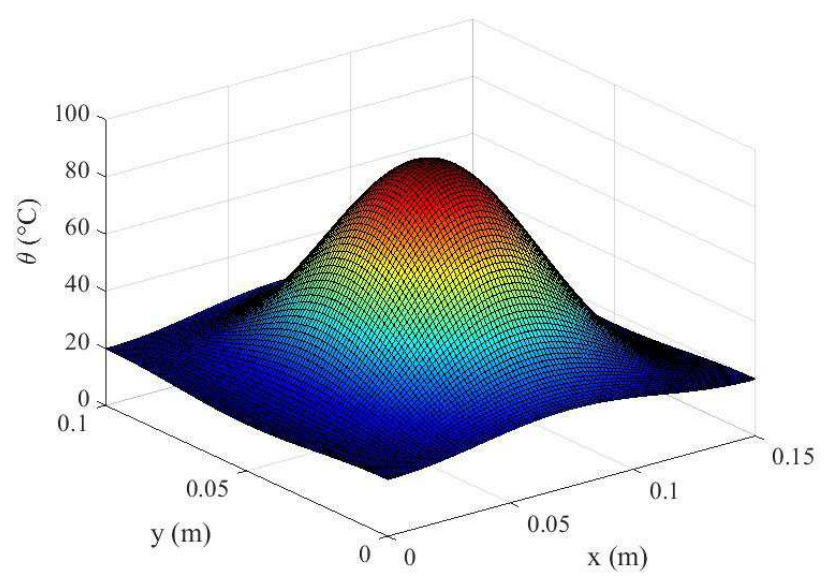

Figure 2. The distribution of the temperature of the plate.

The maximum temperature $\theta_{\max }$ for heating the plate is also reached at this point and is equal to $90.1^{\circ} \mathrm{C}$. The figures show the characteristics calculated for the points of maximum values of amplitudes and temperatures for corresponding vibration modes.

In figures 3 and 4 , the amplitude- and temperaturefrequency responses on vibration at the point of maximum deflections and temperatures in the vicinity of the first resonance frequency under a transverse loading with amplitude $q^{\prime}=400 \mathrm{~Pa}$ for different values of $N_{x x}$ are shown. In figures 3 and 4 , the blue, red and green lines show the amplitude - and temperature-frequency responses for the values of $N_{x x}=0,20$, and $45 \mathrm{kN} / \mathrm{m}$ respectively. 


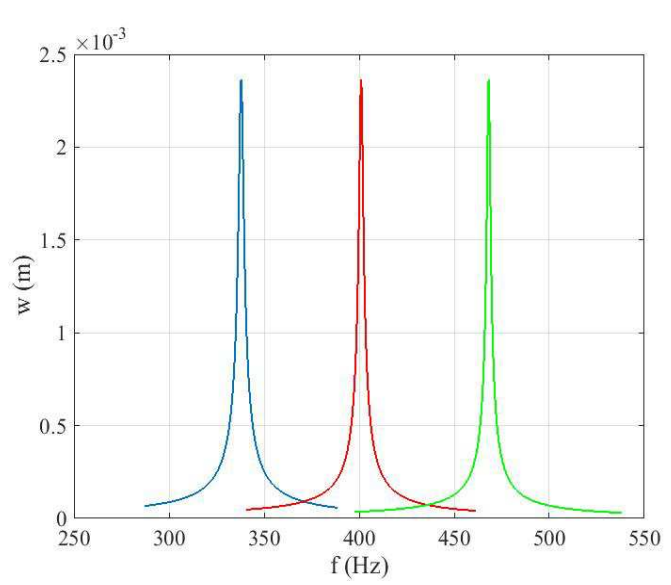

Figure 3. The amplitude-frequency response of the plate.

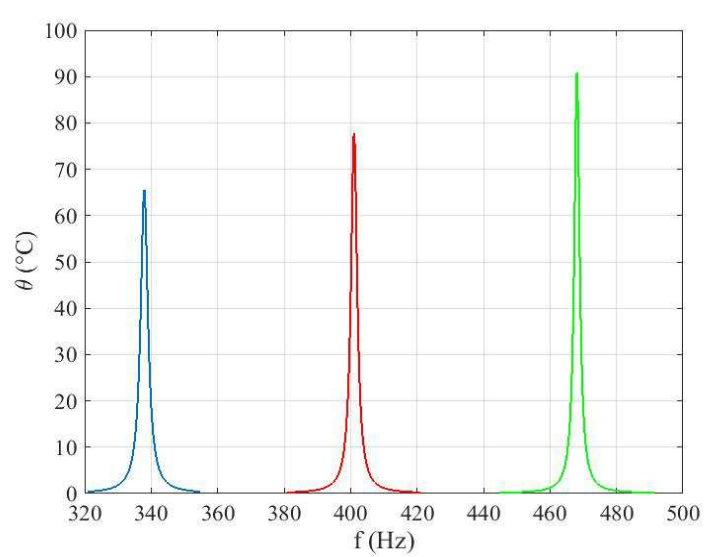

Figure 4. The temperature-frequency response of the plate.

Figure 3 illustrates the effect of shifting the resonant frequency (to the region of higher frequency values) with an increase in preliminary tensile membrane tractions in the plate, which corresponds to the known theoretical and experimentally confirmed result. A similar effect is obtained for the case of temperature frequency response (Figure 4). The shift of the resonance region to the right is accompanied by an increase in temperature.

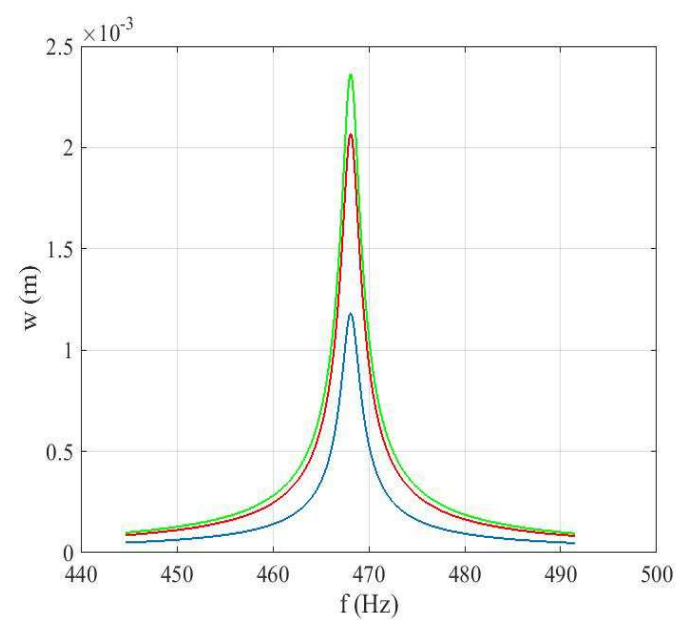

Figure 5. The amplitude-frequency response of the plate under different values.
In figures 5 and 6 , the amplitude- and temperaturefrequency responses on vibration of the square plate in the vicinity of the first resonance frequency for $N_{x x}=45 \mathrm{kN} / \mathrm{m}$ under different values of the transverse loading are shown. In figures 5 and 6 , the blue, red, and green lines show the amplitude- and temperature-frequency responses for the values of transverse loading with amplitudes $q^{\prime}=200,350$, and $400 \mathrm{~Pa}$ respectively.

As it can be seen from figure 5, the behavior of the amplitude-frequency response corresponds to the typical behavior of the characteristics in the case of a linear viscoelastic material and is accompanied by an increase in the amplitude of deflection with an increase in the amplitude of the transverse loading.

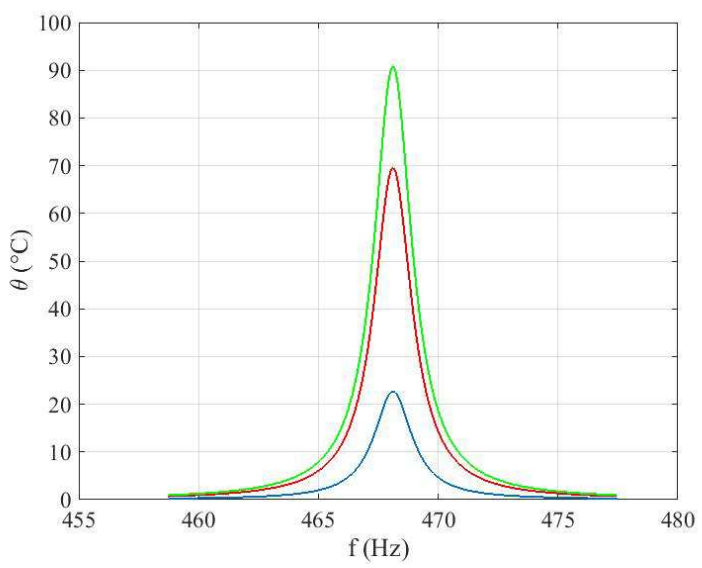

Figure 6. The temperature-frequency response of the plate under different values.

The temperature-frequency response corresponding to this case is presented in figure 6. It illustrates a sharp increase in temperature at resonance frequency, which, a critical value of the loading parameter is reached, leads to dangerous levels of heating. The maximum temperature is equal to $90.1^{\circ} \mathrm{C}$.

For the elastomer chosen as the plate material, the melting point is $120^{\circ} \mathrm{C}$. Therefore, in this work, the value of $100^{\circ} \mathrm{C}$ was chosen as the critical value of the heating temperature $\theta_{c r}$, at which a noticeable softening of the material is observed.

\subsection{Non-stationary Problem}

Next, the non-stationary problem for the square plate was considered. The heat conduction equation (14) complemented with thermal initial and boundary conditions (15) was solved.

The ambient temperature is chosen to be equal to $T_{C}=20^{\circ} \mathrm{C}$. The dissipative heating temperature equals $\theta=T-T_{C}$.

In figure 7 , the temperature-time dependence at the resonant frequency $f=468.1 \mathrm{~Hz}$ for the normal membrane force $N_{x x}=45 \mathrm{kN} / \mathrm{m}$ under different values of the transverse loading is shown. In figure 7, the green, violet, blue, and red lines show the temperature-time dependence for the values of transverse loading with amplitudes $q^{\prime}=200,350,400$, and $500 \mathrm{~Pa}$ respectively. 


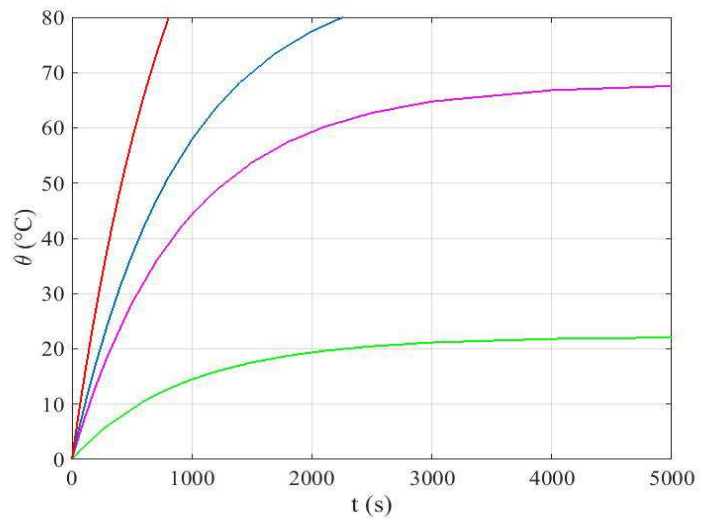

Figure 7. The temperature-time dependence under different values of the transverse loading.

The distribution of the temperature of the plate at point $y=b / 2$ at the resonant frequency $f=468.1 \mathrm{~Hz}$ for the value $N_{x x}=45 \mathrm{kN} / \mathrm{m}$ is shown in the figure 7 .

In figure 8 , the green, violet, blue, and red lines show the distribution of the temperature for the values of transverse loading with amplitudes $q^{\prime}=200,350,400$, and $500 \mathrm{~Pa}$ respectively after 800 seconds of vibration.

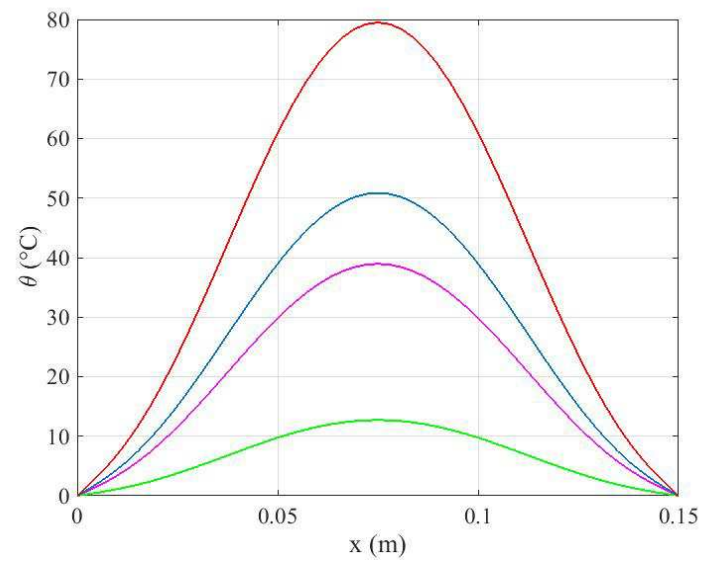

Figure 8. The distribution of the temperature of the plate at point $y=b / 2$.

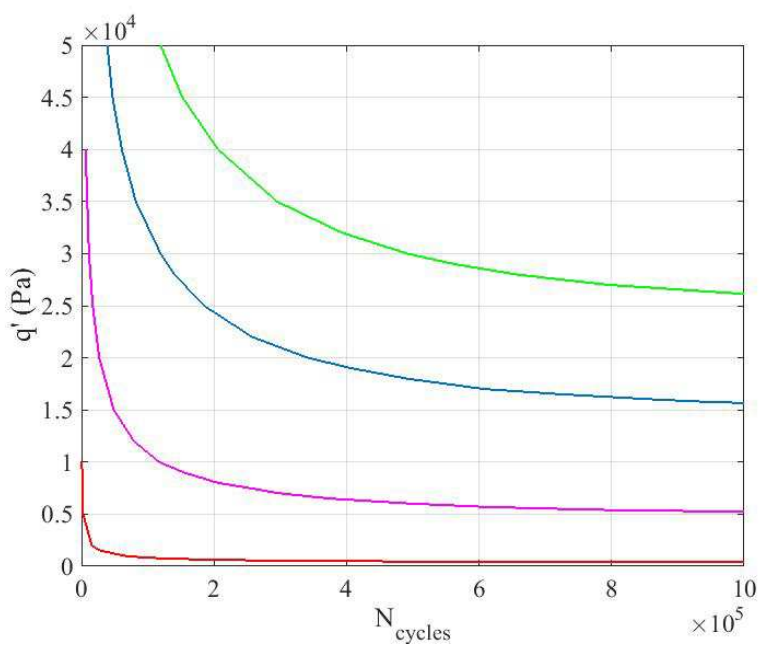

Figure 9. Wöhler diagram calculated at the falling branch of the temperature-frequency characteristics.
Figure 8 illustrates the distribution of the temperature in the first eigenmode $(m=1, n=1)$. The maximum temperature is reached at $x=a / 2$ and equals $\theta_{\max }=79.5^{\circ} \mathrm{C}$.

The first eigenmode is the most energy-intensive vibration mode.

In figure 9, the Wöhler diagram at frequency $f=468.1 \mathrm{~Hz}$, which is resonant for the membrane force $N_{x x}=45 \mathrm{kN} / \mathrm{m}$, was constructed. In figure 9 , the green, blue, violet, and red lines corresponds to $N_{x x}=20,30,40$, and $45 \mathrm{kN} / \mathrm{m}$ respectively. This is called a falling branch.

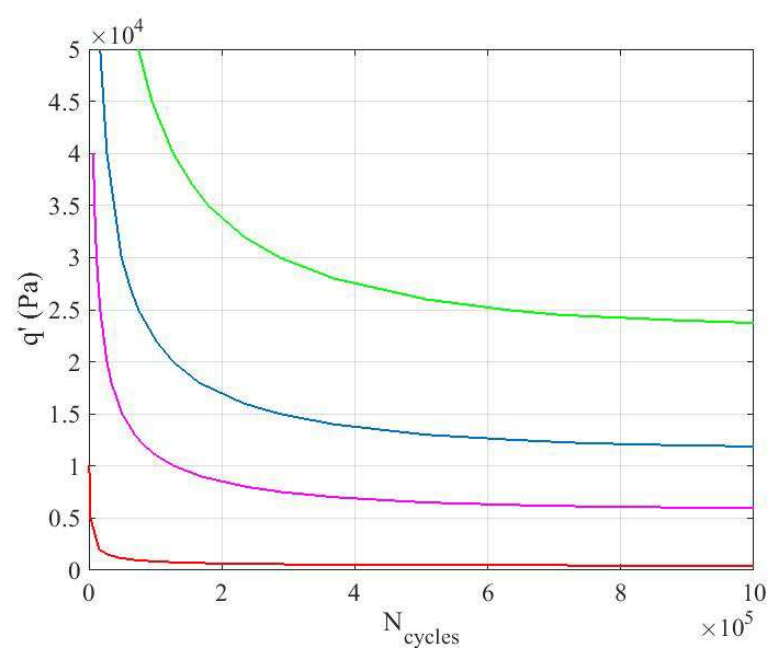

Figure 10. Wöhler diagram calculated at the raising branch of the temperature-frequency characteristics.

In figure 10, the Wöhler diagram at frequency $f=337.9 \mathrm{~Hz}$, which is resonant for the membrane force $N_{x x}=0$, was constructed. In figure 10 , the red, violet, blue, and green lines corresponds to $N_{x x}=0,5,10$, and $20 \mathrm{kN} / \mathrm{m}$ respectively. This is called a raising branch.

To provide the efficiency of the plate, it is necessary to choose such modes on vibration in which the level of heating does not exceed a critical value $\theta_{c r}$. Critical levels of prestresses and loads are determined by the points of intersection of the temperature-frequency response with a line of $\theta_{c r}=100^{\circ} \mathrm{C}$.

\section{Conclusion}

Under the assumption of the Kirchhoff-Love hypotheses validity, the formulation and solution of the stationary and non-stationary problems of forced resonant vibrations and vibroheating of a viscoelastic polyethylene plate with preliminary normal membrane force in case of a rectangular plate, hinged along the contour are presented. For the most energy-intensive first mode of bending vibrations of a pivotally supported plate, the influence of the indicated preliminary state, as well as the level of the transverse distributed load, material properties, heat transfer conditions on the frequency dependences of the maximum deflection amplitude and dissipative heating temperature was studied. The critical values of prestresses are determined at which the 
temperature of the vibroheating reaches a value at which the plate material begins to soften. Temperature-time dependences for a given elastomeric plate are shown. The material fatigue limits are found for which the Wöhler diagrams are constructed.

\section{References}

[1] N. Tasmin, A. T. Salman, R. A. Beg. A study of a Hybrid Heat Pipe Solar Collector with an Absorber Plate. Advances in Applied Sciences. Vol. 4, No. 6, 2019, pp. 110-119. doi: $10.11648 /$ j.aas. 20190406.12

[2] R. Steinberger, T. I. Valadas Leit ao, E. Landstatter, G. Pinter, W. Billinger, R. W. Lang, "Infrared thermographic techniques for non-destructive damage characterization of carbon fibre reinforced polymers during tensile fatigue testing", Int. J. Fatigue 28, 1340 (2006).

[3] D. Rittel, "On the conversion of plastic work to heat during high strain rate deformation of glassy polymers", Mech. Mater. 31, 131 (1999).

[4] S. Moissa, G. Landsberg, D. Rittel, J. L. Halary, "Hysteretic thermal behavior of amorphous semi-aromatic polyamides", Polymer 45, 11870 (2005).

[5] S. Mortazavian., A. Fatemi, "Fatigue behavior and modeling of short fiber reinforced polymer composites: A literature review", Int. J. Fatigue 70, 297 (2015).

[6] R. N. Haward, "Heating effects in the deformation of thermoplastics", Thermochim. Acta 247, 87 (1994).

[7] M. Hashemi, Y. Zhuk, "The influence of strain amplitude, temperature and frequency on complex shear moduli of polymer materials under kinematic harmonic loading", Mech. Mech. Eng. 21, 157 (2017).

[8] M. Mehdizadeh, M. M. Khonsari, "On the application of fracture fatigue entropy to variable frequency and loading amplitude", Theor. Appl. Fract. Mech. 98, 30 (2018).

[9] A. Krairi, I. Doghri, "A thermodynamically-based constitutive model for thermoplastic polymers coupling viscoelasticity, viscoplasticity and ductile damage", Int. J. Plast. 60, 163 (2014).
[10] A. Katunin, M. Fidali, "Fatigue and thermal failure of polymeric composites subjected to cyclic loading", Adv. Compos. Lett. 21, 64 (2012).

[11] S. Mortazavian, A. Fatemi, "Fatigue of short fiber thermoplastic composites: A review of recent experimental results and analysis", Int. J. Fatigue 102, 171 (2017).

[12] A. Katunin. Criticality of the Self-Heating Effect in Polymers and Polymer Matrix Composites during Fatigue, and Their Application in Non-Destructive Testing. Polymers 11, DOI: org/10.3390/polym11010019 (2019).

[13] I. K. Senchenkov, Ya. A. Zhuk, and V. G. Karnaukhov, "Modeling the Thermomechanical Behavior of Physically Nonlinear Materials under Monoharmonic Loading”, Int. Appl. Mech. 40, 943 (2004).

[14] Y. A. Zhuk, I. K. Senchenkov, "On Linearization of the Stiffness Characteristics of Flexible Beams Made of Physically Nonlinear Materials", Int. Appl. Mech. 42, 196 (2006).

[15] Y. A. Zhuk, I. K. Senchenkov, "Monoharmonic approach to investigation of the vibrations and self-heating of thin-wall inelastic members", J. Civil EngrgManag. 15, 67 (2009).

[16] V. G. Karnaukhov, I. F. Kirichok, "Forced Harmonic Vibrations and Dissipative Heating-up of Viscoelastic Thin-Walled Elements (Review)", Int. Appl. Mech. 36, 174 (2000).

[17] M. Hashemi, Y. A. Zhuk, "The Influence of Temperature on the Cyclic Properties of the Transversely Isotropic Nanocomposite System Under Kinematic Harmonic Loading”, J. Mat. Sci. 236, 185 (2019).

[18] Y. A. Zhuk, "Damping characteristics of three-layer beam-damper under harmonic loading", Mat. Mod. Comp. 1, 109 (2014).

[19] Reddy J. N. Theory and Analysis of Elastic Plates and Shell. CRC Press; (2006).

[20] Donnell L. H. Beams, Plates and Shells. McGraw-Hill; (1976).

[21] Lazan B. Damping of materials and members in structural mechanics. Oxford etc., Pergamon Press; (1968). 